\title{
Integer and half-integer flux-quantum transitions in a niobium-iron pnictide loop
}

\author{
C.-T. Chen ${ }^{1 \star}$, C. C. Tsuei ${ }^{1}$, M. B. Ketchen ${ }^{1}$, Z.-A. Ren ${ }^{2}$ and Z. X. Zhao ${ }^{2}$
}

The recent discovery of iron-based superconductors ${ }^{1-3}$ challenges the existing paradigm of high-temperature superconductivity. Owing to their unusual multi-orbital band structure $^{4,5}$, magnetism ${ }^{6}$ and electron correlation ${ }^{7}$, theories propose a unique sign-reversed $s$-wave pairing state, with the order parameter changing sign between the electron and hole Fermi pockets ${ }^{8-14}$. However, because of the complex Fermi surface topology and materials-related issues, the predicted sign reversal remains unconfirmed. Here we report a new phase-sensitive technique for probing unconventional pairing symmetry in the polycrystalline iron pnictides. Through the observation of both integer and half-integer flux-quantum transitions in composite niobium-iron pnictide loops, we provide the first phase-sensitive evidence of the sign change of the order parameter in $\mathrm{NdFeAsO}_{0.88} \mathrm{~F}_{0.12}$, lending strong support for microscopic models predicting unconventional $s$-wave pairing symmetry ${ }^{9-14}$. These findings have important implications on the mechanism of iron pnictide superconductivity, and lay the groundwork for future studies of new physics arising from the exotic order in the FeAs-based superconductors.

The new superconducting $\mathrm{Fe}$ pnictides consist of two key subsets: (1) the one-layer 1111 compounds, $\mathrm{LnFeAsO}_{1-x} \mathrm{~F}_{x}$, where Ln: lanthanides (including $\mathrm{La}, \mathrm{Nd}$ and $\mathrm{Sm}$ ), with a superconducting transition temperature $\left(T_{\mathrm{c}}\right)$ ranging from 26 to $55 \mathrm{~K}$ (refs 1 , 2); (2) the bi-layer 122 compounds, such as $(\mathrm{Ba}, \mathrm{K}) \mathrm{Fe}_{2} \mathrm{As}_{2}$ and $(\mathrm{Sr}, \mathrm{K}) \mathrm{Fe}_{2} \mathrm{As}_{2}$, with a $T_{\mathrm{c}}$ up to $38 \mathrm{~K}$ (ref. 3). Like cuprates, the superconducting phase of Fe pnictides emerges in close proximity to the antiferromagnetic order ${ }^{6}$. In addition, the low-energy electronic states are shown to derive from the conducting FeAs layers $^{4}$ (predominantly of $\mathrm{Fe} 3 d$ character), very much like the $\mathrm{CuO}_{2}$ planes in cuprates. However, striking differences do exist between the two classes of high- $T_{\mathrm{c}}$ materials. For instance, the new Fe pnictide superconductors are characterized by a multiorbital electronic band structure and disconnected Fermi surface sheets $^{4,5}$, in contrast to the cuprates with a single Fermi surface. Furthermore, the onsite Coulomb correlation is considerably weaker ${ }^{7}$. The similarities and disparities between the Fe pnictides and cuprates naturally bring about the question of whether the two share the same pairing mechanism. Determining the symmetry of the pair wavefunction in the new superconductors is thus of great importance for narrowing down possible microscopic theories. The existence of multiple Fermi surface sheets in these materials considerably complicates the investigations of their pairing symmetry, because various intra- and inter-Fermisurface scattering processes can participate in Cooper pairing ${ }^{8-14}$. Present theories of the $\mathrm{Fe}$ pnictides have predicted a variety of superconducting order parameters, from spin-triplet $p$-wave pairing ${ }^{15}$, sign-reversed extended $s$-wave $(s \pm)$ pairing ${ }^{9-14}$ to $d$-wave pairing ${ }^{10,12,16}$. At present, most theories favour $s \pm$ pairing when



Figure 1 | Schematic of the experimental set-up. The composite loop is made of a 0.75-mm-diameter Nb wire (a), polished at both ends, shaped into a 2-mm-diameter ring and pressed against the polycrystalline $\mathrm{Nd}-1111$ sample (b) to form a complete circuit. Three $\mathrm{Cu}$-Be spring-loaded brass screws (c) control the contact strength between the sample and the $\mathrm{Nb}$ tips. The distance from the centre of the loop to the contact points is $\sim 7.5 \mathrm{~mm}$. The 1.6-mm-diameter Nb toroidal coil (d) applies magnetic flux to the loop from a low-noise current source by means of a twisted pair of leads. The Nb pickup coil (e) couples the induced flux to a commercial d.c.-SQUID (Quantum Design Model 50) as a magnetometer to monitor the flux states of the loop. The Nb solenoid ( $f$ ) coupled to the composite loop transmits electromagnetic pulses from the external world to induce transitions between different quantized flux states.

realistic material parameters are considered ${ }^{9-14}$. However, there is still no definitive experimental confirmation.

Here we present such an experiment designed to probe the predicted sign change of the pair wavefunction in the Fe pnictide superconductors. Until now, most pairing symmetry experiments have focused on the 122-type Fe pnictide compounds ${ }^{17,18}$. In the case of the 1111-type superconductors, besides the complications of the Fermi surface topology, the lack of high-quality epitaxial films and large single crystals has impeded standard phasesensitive pairing tests ${ }^{19}$. Almost all of the pairing experiments done on this class of materials are only amplitude sensitive ${ }^{8}$, except for the scanning superconducting quantum interference device (SQUID) measurements ${ }^{20}$. Be they thermodynamic ${ }^{21-23}$ or tunnelling-based ${ }^{24}$, these measurements are prone to impurity

${ }^{1}$ IBM Thomas J. Watson Research Center, Yorktown Heights, New York 10598, USA, ${ }^{2}$ Institute of Physics and Beijing National Laboratory for Condensed Matter Physics, Chinese Academy of Sciences, Beijing 100190, China. *e-mail: cchen3@us.ibm.com. 
a

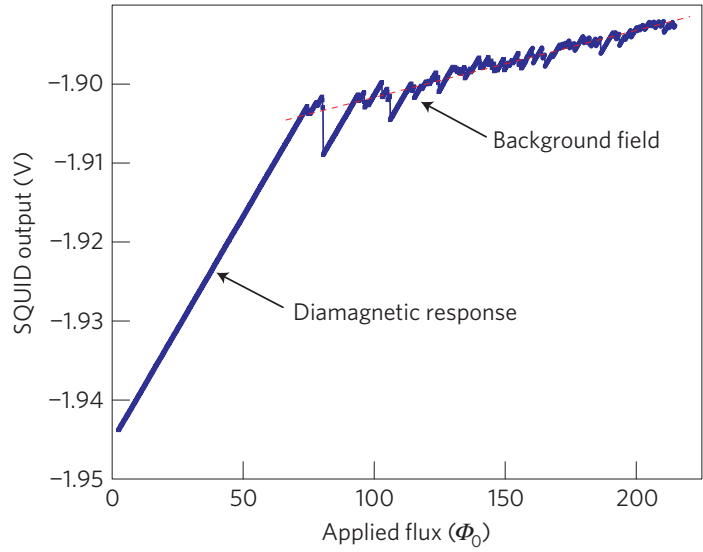

b

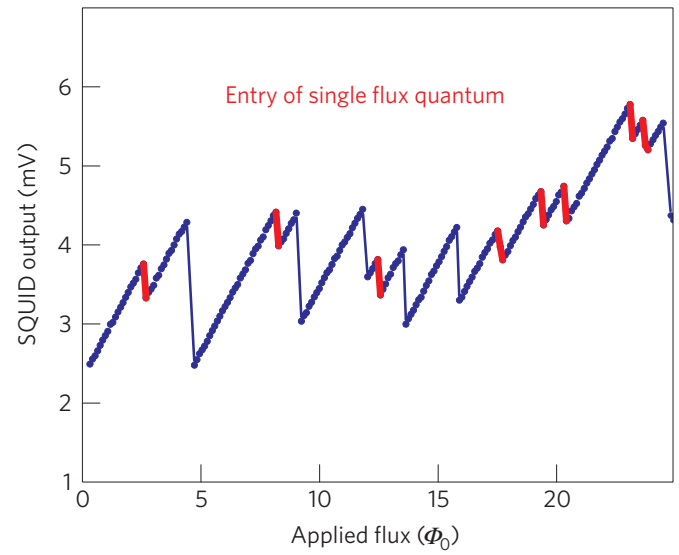

Figure 2 | Induced flux of the composite $\mathrm{Nb}-\mathrm{NdFeAsO} \mathrm{O}_{0.88} \mathrm{~F}_{0.12}$ loop with applied flux. The horizontal axis plots the applied magnetic flux converted from the toroidal coil current. The vertical axis plots the induced flux detected by the SQUID. a, Typical diamagnetic response of a composite loop with a junction critical current $I_{c} \sim 50 \mu \mathrm{A}$. Along the sloping section, the induced supercurrent cancels out the applied flux completely to ensure flux conservation. When the applied flux is kept constant, the induced current remains at a constant value with no measurable decay, indicative of a loop resistance $\ll 10^{-13} \Omega$. We note that when the induced supercurrent exceeds $I_{c}$, there remains a small residual slope in the SQUID output (see thin dashed line for a linear fit) resulting from the coupling of the SQUID pickup coil to the small leakage field of the toroidal coil. $\mathbf{b}$, Typical response of a composite loop with $I_{\mathrm{c}} \sim 5 \mu \mathrm{A}$. Single and integer-multiple flux-quantum entries on increasing applied flux are clearly resolved. The red segments highlight single-quantum jumps and the blue jumps describe entries of multiple flux quanta (from left to right, 4, 3, 2, 2 and $3 \Phi_{0}$ ).

contamination, surface degradation or complications introduced by parameter fitting ${ }^{8,19}$. Our approach circumvents these problems by resorting to the integrity of the quantum phase in a composite $\mathrm{Nb}-\mathrm{NdFeAsO} \mathrm{O}_{0.88} \mathrm{~F}_{0.12}$ (Nb-Nd-1111) superconducting loop. We first establish supercurrent and flux quantization in the composite loop. Then, by inducing and detecting half-flux-quantum transitions in the loop, we establish the first phase-sensitive evidence for the sign change in the Fe pnictide order parameter.

Figure 1 illustrates the experimental set-up for observing persistent current and flux quantization. (Details of the sample preparation and characterization are given in the Supplementary Information.) Our method is inspired by similar experiments on yttrium barium copper oxide in the early days of cuprate superconductivity research ${ }^{25}$, yet with a greater flexibility in sample exchange that enables precise calibration of the background field and against known samples, such as $\mathrm{Nb}$ (see Supplementary Methods). The components enclosed in the dashed red square in



Figure 3 | Integer flux-quantum jumps in the composite

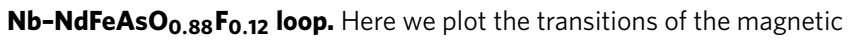
flux states of the composite loop on pulsed electromagnetic excitations in the weak-coupling limit $\left(I_{\mathrm{c}}\right.$ below $\left.\sim 0.5 \mathrm{~mA}\right)$. Three runs of inter-flux-state transition measurements have been recorded, with contacts formed at different locations on the sample surface and of varying loop critical currents in the submilliampere range. After calibrating against the standard $\mathrm{Nb}-\mathrm{Nb}$ loop, the unit of a flux quantum jump in the Nb-Nd-1111 loop is determined to be $\sim(1.02 \pm 0.03) \Phi_{0}$, taking into account the systematic error and the statistical variations.

Fig. 1 are immersed in liquid helium with an ambient magnetic field below $50 \mu \mathrm{G}$. The low residual field is achieved by placing four layers of $\mu$-metal shield around the Dewar and two layers of superconducting $\mathrm{Pb}$ foil around the sample box. The entire system further sits in a shield room to guard against external radiofrequency contamination. An ambient white-noise level of $\leq 6 \mu \Phi_{0} / \sqrt{\mathrm{Hz}}$ at $\sim 1 \mathrm{~Hz}$ measured at the d.c.-SQUID is routinely obtained, where the flux quantum

$$
\Phi_{0}=h / 2 e=2.07 \times 10^{-7} \mathrm{G} \mathrm{cm}^{2}
$$

Figure 2 shows the SQUID readout of the induced flux as a function of the toroidal coil current, expressed in terms of the applied magnetic flux. To conserve total flux, on ramping up the coil current, supercurrent is induced to cancel out the applied flux threading through the $\mathrm{Nb}-\mathrm{Nd}-1111$ loop. When the induced supercurrent finally exceeds the critical current of the weakest junction in the loop, magnetic flux starts to move in or out of the loop in single or multiple units of $\Phi_{0}$, giving rise to the zigzag pattern in the field sweep (Fig. 2a). As we decrease the contact strength so that the junction critical current $I_{\mathrm{c}}$ drops below $\sim 20 \mu \mathrm{A}$, unambiguous features corresponding to single flux-quantum entries become well resolved (Fig. 2b). The persistent current in the loop implies that macroscopic quantum coherence is established across the $\mathrm{Nb}-\mathrm{Nd}-1111$ junctions. The observation of single flux-quantum entry clearly indicates that the enclosed flux is quantized and that the phase of the pair wavefunction is coherent throughout the entire loop.

To provide direct evidence of flux quantization, we present SQUID measurements of the transitions between different flux quantum states in zero fields triggered by the intermittently applied electromagnetic pulses (Fig. 3). On exposure to the electromagnetic disturbance, magnetic flux enters or exits the loop in quantized steps, as illustrated by the equally spaced grid lines in Fig. 3. Through comparisons with the SQUID sensitivity experiment (Supplementary Fig. S2) and the magnitude of flux jumps in the standard calibration $\mathrm{Nb}-\mathrm{Nb}$ loop (Supplementary Fig. S3), we determine the magnitude of a flux quantum jump in the $\mathrm{Nb}-\mathrm{Nd}-1111$ loop to be $\sim(1.02 \pm 0.03) \Phi_{0}$ (see Supplementary 


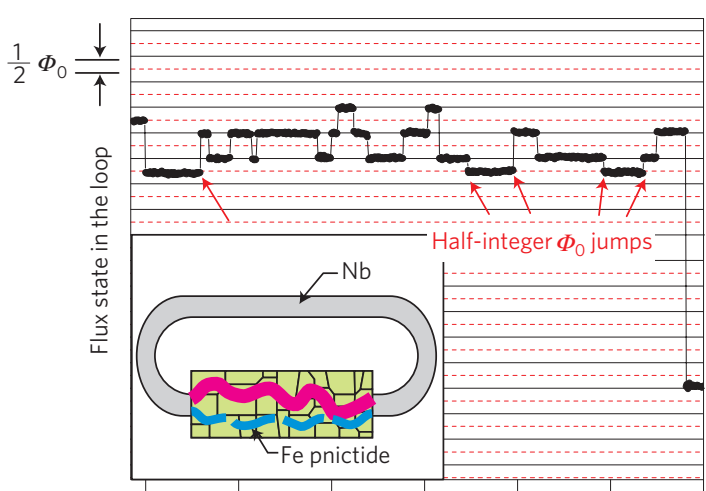

b

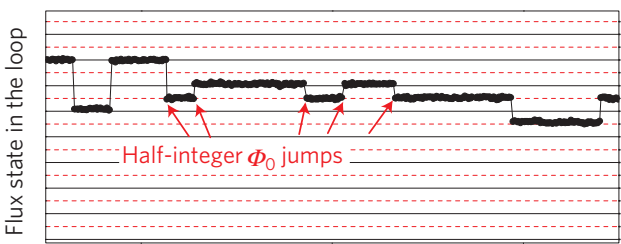

c

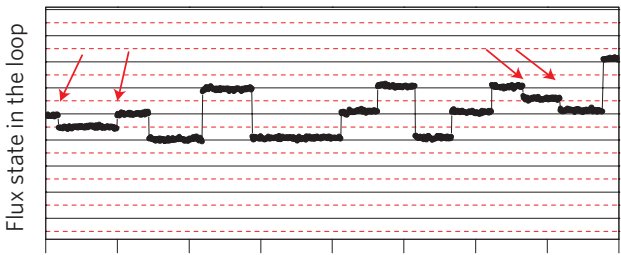

d

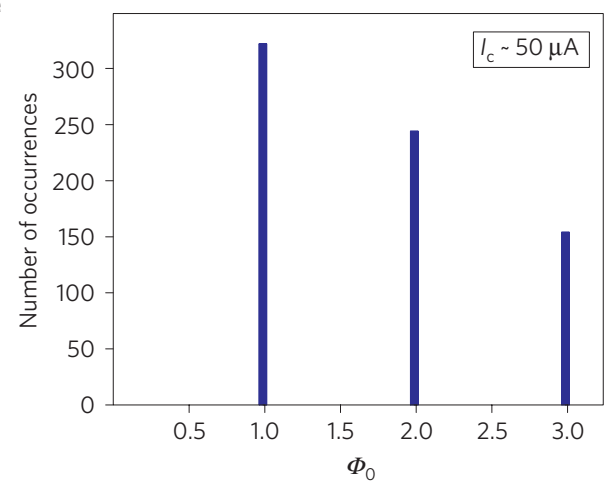

f

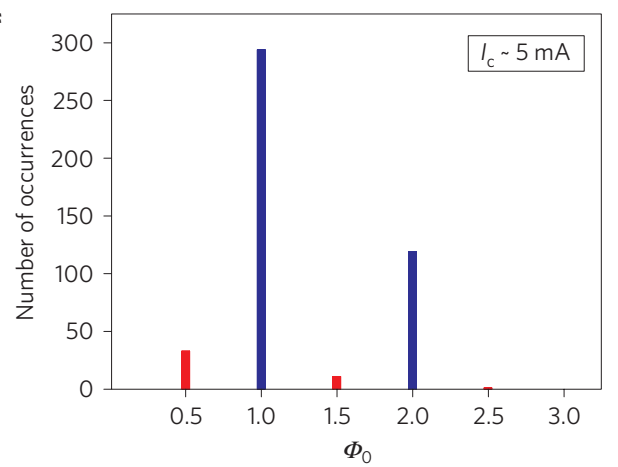

g

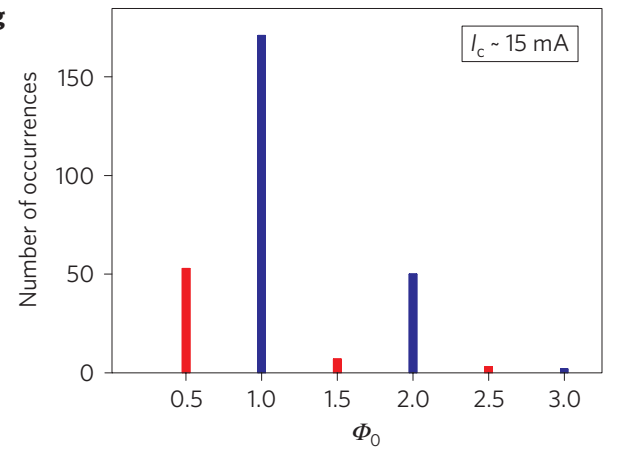

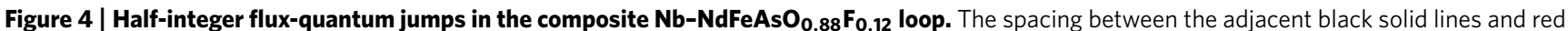
dashed lines is $1 / 2 \Phi_{0}$. The time interval between two ticks is $50 \mathrm{~s}$. The red arrows label the half-integer flux-quantum jumps. a,b, Representative data taken on a composite loop with $I_{\mathrm{c}} \sim 5 \mathrm{~mA}$. The inset of a illustrates schematically that the supercurrent distribution in the composite loop passes through a large number of grain-boundary junctions with a wide range of incident angles in the polycrystalline $\mathrm{NdFeAsO}_{0.88} \mathrm{~F}_{0.12}$. The realistic current flow pattern is much more complicated than depicted. $\mathbf{c}, \mathbf{d}$, Representative data taken on a composite loop with $I_{\mathrm{c}} \sim 15 \mathrm{~mA}$. Three sets of half-integer-transition data have been recorded with contacts formed at different locations on the sample surface and of varying $I_{\mathrm{c}}$ in the milliampere range. e- $\mathbf{g}$, Histograms of integer versus half-integer transitions on pulsed electromagnetic excitations. We note that the width of each bin does not reflect the error bar of the measured flux jumps (which is about $\pm 0.03 \Phi_{0}$ ).

Methods). As a sanity check, the same set of measurements is repeated in the open loop configuration where the junction resistance is infinite. We verify that when the circuit is open, the residual slope in the field-sweep response is consistent with the background observed in Fig. 2 and that there is no observable flux jump.

As demonstrated in Figs 2 and 3, in a weak-coupling composite ring ( $I_{\mathrm{c}}$ below $\sim 0.5 \mathrm{~mA}$ ), magnetic fluxons escape or enter the loop readily at the $\mathrm{Nb}-\mathrm{Nd}-1111$ junction interface by a phase slip of $2 n \pi$ ( $n$ is an integer), giving rise to the observed integer fluxquantum transitions. However, there is a distinct possibility that half-integer flux quantization can be observed in our $\mathrm{Nb}-\mathrm{Nd}-1111$ ring experiment with increasing $I_{\mathrm{c}}$, if the polycrystalline Nd-1111 has a sign change (that is, a $\pi$ phase shift) in the superconducting order parameter and if its crystallites are properly oriented to form a $\pi$-loop. Consider a $\mathrm{Nb}-\mathrm{Fe}$ pnictide composite loop as shown in the inset of Fig. 4a. According to refs 26 and 27, a phase shift of $\pi$ in tunnelling could occur at the Nb-Nd-1111 interface. Besides, intergrain transport between electron and hole pockets may also give rise to a $\pi$ shift, although this is much less likely ${ }^{8}$. Along the current path with $\mathrm{Nb}-\mathrm{Nd}-1111$ contact areas up to $\sim 500 \mu \mathrm{m}$ in diameter (when $I_{\mathrm{c}}$ is increased to the milliampere range) and tip-tip separation $\sim 500 \mu \mathrm{m}$, at least hundreds of junctions are involved, and a wide range of tunnelling direction is sampled. All of these significantly enhance the chance of forming $\pi$-shifted junctions. At zero magnetic field, under the experimental condition of $\left|I_{\mathrm{c}}\right| L \gg \Phi_{0}$, where $L(\sim 5 \mathrm{nH})$ is the self-inductance of the loop, the enclosed magnetic flux can be described by

$$
\begin{gathered}
\Phi=n \Phi_{0}, \quad \text { for } N=\text { even }(0 \text {-loop }) \\
\Phi=(n+1 / 2) \Phi_{0}, \quad \text { for } N=\text { odd }(\pi \text {-loop })
\end{gathered}
$$


where $N$ is the number of $\pi$ phase shifts around the circulating supercurrent loop ${ }^{19}$. The half-flux-quantum becomes observable in our experiment when the large $\mathrm{Nb}$ loop is terminated by a Josephson junction array containing $\pi$ phase shifts. In a simple model of this composite loop with a small-inductance parallel $0-\pi$ junction circuit formed at the $\mathrm{Nb}-\mathrm{Nd}-1111$ interface or within the sample, the half-integer flux quantum is manifested in the transitions between the meta-stable quantized flux states in the big Nb loop.

We look for the signatures of such a half-flux-quantum effect by forcing the current distribution to switch between the two local-energy-minimum configurations. Towards this end, we use a tighter coupling between the loop and the intermittently applied electromagnetic pulses. The stronger pulsed electromagnetic disturbance induces a surge in diamagnetic transient supercurrent that produces the driving force to propagate the half-flux-quantum across the $0-\pi$ junction array ${ }^{28,29}$ into the $\mathrm{Nb}$ loop, leading to half-flux-quantum transitions and supercurrent redistribution in the composite circuit. At the same time, we increase the contact strength to bring more Nd-1111 crystal grains in contact with the $\mathrm{Nb}$ tips and enhance the Josephson coupling at the Nb-Nd-1111 interface. This amplifies the probability of forming a $\pi$-shifted junction ${ }^{26,27}$ and suppresses the integer fluxquantum entries across the contact, leading to a higher occurrence of the half-flux-quantum entries.

When $I_{\mathrm{c}}$ is increased to $\sim 5 \mathrm{~mA}$, clear signals of half-integer flux-quantum jumps begin to show up on electromagnetic exposure (see Fig. 4a,b). Four typical time traces of the transitions are shown in Fig. 4a-d. Changes of flux states between a black grid line and a red dashed line correspond to half-integer flux-quantum jumps. Examples of such events are pointed out with the red arrows. As shown in Fig. 4e-g, a total of more than one hundred half-integer transitions were recorded. No other fractional steps were observed. Taking into account the environmental noises and drifting, we estimate that the measured half-integer jumps are accurate to $(0.51 \pm 0.03) \Phi_{0}$. The histograms (Fig. $4 \mathrm{e}-\mathrm{g}$ ) clearly demonstrate that the probability of observing a half-flux-quantum transition is larger for a composite loop with a higher critical current, which is consistent with the scenario of forming more $\pi$-phase-shifted junctions at the $\mathrm{Nb}-\mathrm{Nd}-1111$ contacts $^{26,27}$. In addition, the distinctly different distribution profiles of the integer and half-integer jumps further corroborate our conjecture that they are driven by two separate physical mechanisms: the integer jumps result from the phaseslip events at the weakest contact junction, whereas the halfinteger jumps originate from the electromagnetic-induced halfflux-quantum propagation ${ }^{28,29}$. Using the same experimental setup, no half-flux-quantum jump in the $\mathrm{Nb}-\mathrm{Nb}$ composite loop was detected in repeated measurements. This null result rules out incidental vortex trapping as the source of the observed $\Phi=(n+1 / 2) \Phi_{0}$ jumps and reconfirms that without any $\pi$-shifted junctions, half-flux-quantum transitions can never occur. We further note that superconductor-ferromagnet-superconductor junctions ${ }^{30}$ are unlikely to be the source of the observed $\pi$ phase shift (see Supplementary Information for a discussion on superconductor-ferromagnet-superconductor junctions and other unlikely alternative sources). Therefore, the presence of half-fluxquantum jumps in the $\mathrm{Nb}-\mathrm{NdFe} A s \mathrm{O}_{0.88} \mathrm{~F}_{0.12}$ loop is direct proof of the sign change in the order parameter of Nd-1111.

The results of our experiment are consistent with $s \pm$ and $d$-wave pairing because triplet-pairing, such as $p$-wave ${ }^{15}$, has been ruled out by NMR experiments ${ }^{23}$. Among these two possibilities, $d$-wave pairing has been proven unlikely in the hole-doped 122 compounds by the $c$-axis tunnelling experiments ${ }^{17}$. Furthermore, in $d$-wave cuprate superconductors, a measurable paramagnetic Meissner effect is known to occur in the polycrystalline samples ${ }^{19}$. A recent scanning SQUID microscopy study on a Nd-1111 sample ${ }^{20}$ almost identical to ours also provides evidence against $d$-wave pairing by the absence of such an effect. (See ref. 8 and Supplementary Information for a discussion on the absence of a paramagnetic Meissner signal in Nd-1111 and $s \pm$ pairing.) As the aforementioned two experiments ${ }^{17,20}$ failed to capture the $\pi$ phase shift, our observation of the half-flux-quantum effect provides much needed phase-sensitive evidence for the sign change, strongly supportive of $s \pm$ pairing in the 1111-type Fe pnictide compounds.

Received 6 November 2009; accepted 21 January 2010; published online 21 February 2010

\section{References}

1. Kamihara, Y., Watanabe, T., Hirano, M. \& Hosono, H. Iron-based layered superconductor $\mathrm{La}\left[\mathrm{O}_{1-x} \mathrm{~F}_{x}\right] \mathrm{FeAs}(x=0.05-0.12)$ with $T_{\mathrm{c}}=26 \mathrm{~K}$. J. Am. Chem. Soc. 130, 3296-3297 (2008).

2. Ren, Z.-A. et al. Superconductivity in iron-based F-doped layered quaternary compound $\mathrm{NdO}_{1-x} \mathrm{~F}_{x}$ FeAs. Europhys. Lett. 82, 57002 (2008).

3. Rotter, M., Tegel, M. \& Johrendt, D. Superconductivity at $38 \mathrm{~K}$ in the iron arsenide $\left(\mathrm{Ba}_{1-x} \mathrm{~K}_{x}\right) \mathrm{Fe}_{2} \mathrm{As}_{2}$. Phys. Rev. Lett. 101, 107006 (2008).

4. Singh, D. J. \& Du, M. H. Density functional study of $\mathrm{LaFeAsO}_{1-x} \mathrm{~F}_{x}$ : A low carrier density superconductor near itinerant magnetism. Phys. Rev. Lett. 100, 237003 (2008)

5. Ding, H. et al. Observation of Fermi-surface-dependent nodeless superconducting gaps in $\mathrm{Ba}_{0.6} \mathrm{~K}_{0.4} \mathrm{Fe}_{2} \mathrm{As}_{2}$. Europhys. Lett. 83, 47001 (2008).

6. de la Cruz, C. et al. Magnetic order close to superconductivity in the iron-based layered $\mathrm{LaO}_{1-x} \mathrm{~F}_{x} \mathrm{FeAs}$ systems. Nature 453, 899-902 (2008).

7. Yang, W. L. et al. Evidence for weak electronic correlations in iron pnictides. Phys. Rev. B 80, 014508 (2009).

8. Mazin, I. I. \& Schmalian, J. Pairing symmetry and pairing state in ferropnictides: Theoretical overview. Physica C 469, 614-627 (2009).

9. Mazin, I. I., Singh, D. J., Johannes, M. D. \& Du, H. M. Unconventional superconductivity with a sign reversal in the order parameter of $\mathrm{LaFeAsO}_{1-x} \mathrm{~F}_{x}$. Phys. Rev. Lett. 101, 057003 (2008).

10. Kuroki, K. et al. Unconventional pairing originating from the disconnected Fermi surfaces of superconducting $\mathrm{LaFeAsO}_{1-x} \mathrm{~F}_{x}$. Phys. Rev. Lett. 101, 087004 (2008)

11. Chen, W.-Q., Yang, K.-Y., Zhou, Y. \& Zhang, F.-C. Strong coupling theory for superconducting iron pnictides. Phys. Rev. Lett. 102, 047006 (2008).

12. Graser, S., Maier, T. A., Hirschfeld, P. J. \& Scalpino, D. J. Near-degeneracy of several pairing channels in multiorbital models for the Fe pnictides. New J. Phys. 11, 025016 (2009).

13. Wang, F., Zhai, H., Ran, Y., Vishwanath, A. \& Lee, D.-H. Functional renormalization-group study of the pairing symmetry and pairing mechanism of the FeAs-based high-temperature superconductor. Phys. Rev. Lett. 102, 047005 (2009)

14. Seo, K., Bernevig, B. A. \& Hu, J. Pairing symmetry in a two-orbital exchange coupling model of oxypnictides. Phys. Rev. Lett. 101, 206404 (2008)

15. Lee, P. A. \& Wen, X. G. Spin-triplet $p$-wave pairing in a three-orbital model for iron pnictide superconductors. Phys. Rev. B 78, 144517 (2008).

16. $\mathrm{Si}, \mathrm{Q}$. \& Abrahams, E. Strong correlations in magnetic frustration in the high $T_{\mathrm{c}}$ iron pnictides. Phys. Rev. Lett. 101, 076401 (2008).

17. Zhang, X. et al. Observation of the Josephson effect in $\mathrm{Pb} / \mathrm{Ba}_{1-x} \mathrm{~K}_{x} \mathrm{Fe}_{2} \mathrm{As}_{2}$ single crystal junctions. Phys. Rev. Lett. 102, 147002 (2009).

18. Christianson, A. D. et al. Unconventional superconductivity in $\mathrm{Ba}_{0.6} \mathrm{~K}_{0.4} \mathrm{Fe}_{2} \mathrm{As}_{2}$ from inelastic neutron scattering. Nature 456, 930-932 (2008).

19. Tsuei, C. C. \& Kirtley, J. R. Pairing symmetry in cuprate superconductors. Rev. Mod. Phys. 72, 969-1016 (2000).

20. Hicks, C. W. et al. Limits on the superconducting order parameter in $\mathrm{NdFeAsO}_{1-x} \mathrm{~F}_{y}$ from scanning SQUID microscopy. J. Phys. Soc. Jpn 78, 013708 (2009)

21. Martin, C. et al. Non-exponential London penetration depth in $\mathrm{RFeAsO}_{0.9} \mathrm{~F}_{0.1}$ ( $\mathrm{R}=\mathrm{La}, \mathrm{Nd}$ ) single crystals. Phys. Rev. Lett. 102, 247002 (2009).

22. Hashimito, K. et al. Microwave penetration depth and quasiparticle conductivity of $\mathrm{PrFeAsO}_{1-y}$ single crystals: Evidence for a full-gap superconductor. Phys. Rev. Lett. 102, 017002 (2009).

23. Nakai, Y., Ishida, K., Kamihara, Y., Hirano, M. \& Hosono, H. S. Evolution from itinerant antiferromagnet to unconventional superconductor with fluorine doping in La FeAs $\left(\mathrm{O}_{1-x} \mathrm{~F}_{x}\right)$ revealed by ${ }^{75} \mathrm{As}$ and ${ }^{139} \mathrm{La}$ nuclear magnetic resonance. J. Phys. Soc. Jpn 77, 073701 (2008).

24. Chen, T. Y., Tesanovic, Z., Liu, R. H., Chen, X. H. \& Chien, C. L. The BCS-like gap in superconductor $\mathrm{SmFeAsO}_{0.85} \mathrm{~F}_{0.15}$. Nature 453, 1224-1227 (2008).

25. Gough, C. E. et al. Flux quantization in a high- $T_{\mathrm{c}}$ superconductor. Nature 326, 835 (1987).

26. Chen, W. Q., Ma, F., Liu, Z. Y. \& Zhang, F. C. $\pi$-junction to probe antiphase $s$-wave pairing in iron pnictide superconductors. Phys. Rev. Lett. 103, 207001 (2009). 
27. Parker, D. \& Mazin, I. I. Possible phase-sensitive tests of pairing symmetry in pnictide superconductors. Phys. Rev. Lett. 102, 227007 (2009).

28. Lazarides, N. Mobile $\pi$-links and half-integer zero-field-like steps in highly discrete alternation $0-\pi$ Josephson junction arrays. Supercond. Sci. Technol. 21 , 045003 (2008).

29. Chandran, M. \& Kulkarni, R. V. Fractionalization of a flux quantum in a one-dimensional parallel Josephson junction array with alternating $\pi$ junctions. Phys. Rev. B 68, 104505 (2003).

30. Ryazanov, V. V. et al. Coupling of two superconductors through a ferromagnet: Evidence for a $\pi$ junction. Phys. Rev. Lett. 86, 2427-2430 (2001).

\section{Acknowledgements}

We are grateful to A. Brinkman, A. A. Golubov, H. Hilgenkamp, D.-H. Lee, K. A. Moler D. M. Newns, J. Z. Sun and F.-C. Zhang for stimulating discussions. We thank
J. R. Rozen for technical assistance with SQUID measurements. We thank the members in Z.X.Z.'s group for assistance with sample preparation and characterization.

\section{Author contributions}

C.-T.C., C.C.T. and M.B.K. designed and coordinated the experiments. C.-T.C. and C.C.T. implemented the measurements, analysed the data and drafted the manuscript. The polycrystalline $\mathrm{NdFeAsO}_{0.88} \mathrm{~F}_{0.12}$ samples were provided by Z.-A.R. and Z.X.Z.

\section{Additional information}

The authors declare no competing financial interests. Supplementary information accompanies this paper on www.nature.com/naturephysics. Reprints and permissions information is available online at http://npg.nature.com/reprintsandpermissions. Correspondence and requests for materials should be addressed to C.-T.C. 